\title{
DIMETILACETAMIDA ASSOCIADA OU NÃO AO GLICEROL PARA CRIOPRESERVAÇÃO DE SÊMEN OVINO
}

\section{DIMETHYLACETAMIDE ASSOCIATED OR NOT TO GLYCEROL FOR CRIOPRESERVATION OF SHEEP SEMEN}

\author{
Gabriel Felipe Oliveira Menezes ${ }^{1}$ \\ Rodrigo Freitas Bittencourt ${ }^{1^{*}}$ \\ Antônio de Lisboa Ribeiro Filho ${ }^{1}$ \\ Fernando de Lima Cardoso ${ }^{1}$ \\ Mariana Alves Andrade Silva ${ }^{1}$ \\ Elisiane Sateles Santos ${ }^{1}$ \\ 1Universidade Federal da Bahia, Salvador, BA, Brasil. \\ *Autor para correspondência - rfb@ufba.br
}

\section{Resumo}

O objetivo do presente estudo foi testar a dimetilacetamida (DMA) em diferentes concentrações, associada ou não ao glicerol (GL), sobre a viabilidade espermática do sêmen ovino congelado. Foram utilizados 10 ejaculados de dois carneiros adultos da raça Santa Inês. Os ejaculados foram divididos em sete grupos experimentais, respeitando o limite máximo de 5\% de DMA, sendo eles: GL6\%, DMA3\%， GL5\%+DMA1\%， GL4\%+DMA2\%， GL3\%+DMA3\%， GL2\%+DMA4\%, GL1\%+DMA5\%. Os espermatozoides criopreservados nos diferentes tratamentos foram analisados quanto à cinética subjetiva, integridade estrutural da membrana plasmática (EOS), integridade funcional da membrana plasmática (CO) e morfologia espermática, observando defeitos totais (DT) e defeitos maiores (DM). A motilidade total (MT) e a progressiva (MP) pós-descongelação nos grupos GL5\%+DMA1\%; GL4\%+DMA2\% e GL3\%+DMA3\%, foram semelhantes $(\mathrm{P}>0,05)$ ao tratamento controle (GL6\%). Destes, o diluidor GL4\%+DMA2\% foi o único que promoveu a manutenção da MT e MP pós-descongelação, quando comparado com o sêmen in natura $(\mathrm{P}>0,05)$. Não foram observadas diferenças significativas $(\mathrm{P}>0,05)$ para os parâmetros de EOS, CO, DT e DM nos diferentes grupos avaliados. A dimetilacetamida associada ao glicerol mostrou-se eficaz na manutenção da viabilidade espermática em ovinos, avaliada pós-descongelação. Entretanto, foi observado efeito deletério da DMA nas concentrações mais elevadas ou quando não esteve associada ao glicerol.

Palavras-chave: criopreservação; dimetilacetamida; ovino; sêmen.

\begin{abstract}
The objective of the present study was to test dimethylacetamide (DMA) at different concentrations, associated or not to glycerol (GL), on the sperm viability of frozen sheep semen. Ten ejaculates of two adult sheep of Santa Ines breed were used. The ejaculates were divided into seven experimental
\end{abstract}


groups, respecting the maximum limit of $5 \%$ of DMA: GL6\%, DMA3\%, GL5\%+DMA1\%, GL4\%+DMA2\%, GL3\%+DMA3\%，GL2\%+DMA4\%， and GL1\%+DMA5\%. The sperm cryopreserved in the different treatments was analyzed based on the subjective kinetic, structural integrity of the plasma membrane (EOS), functional integrity of the plasma membrane (OS) and sperm morphology, observing total defects (TD) and major defects (MD). The post-thawed total mortality (TM) and progressive mortality (PM) in the GL5\%+DMA1\% groups; GL4\%+DMA2\% and GL3\%+DMA3\% were similar ( $>0.05)$ to the control treatment (GL6\%). Of these, the diluent GL4\%+DMA2\% was the only one that promoted the maintenance of post-thawed TM and PM when compared to in natura semen $(\mathrm{P}>0.05)$. No significant differences $(\mathrm{P}>0.05)$ were observed for the EOS, OS, TD and MD parameters, in the different groups evaluated. Dimethylacetamide associated to glycerol were effective in maintaining sperm viability in post-thawed sheep semen. However, a deleterious effect of DMA was observed at the highest concentrations or when it was not associated with glycerol.

Keywords: cryopreservation; dimethylacetamide; semen; sheep.

Recebido em: 26 de julho de 2017.

Aceiro em: 11 de maio de 2018.

\section{Introdução}

Com o aumento do interesse comercial pela utilização da inseminação artificial (IA) nos programas de melhoramento genético, tem-se buscado o desenvolvimento de diluidores seminais melhorados ${ }^{(1)}$, os quais possibilitam o armazenamento do sêmen de animais por tempo indeterminado ${ }^{(2)}$, além de promover e difundir material genético de animais com alto valor zootécnico ${ }^{(3,4)}$. Entretanto, este é um processo complexo que envolve o equilíbrio de inúmeros fatores para obtenção de resultados satisfatórios $^{(5,6)}$.

Diversos estudos têm sido realizados na tentativa de desenvolver um meio diluidor adequado para o congelamento do espermatozoide ovino. Para Salamon e Maxwell ${ }^{(2)}$, é necessário que os diluentes utilizados na conservação de sêmen ovino, bem como para outras espécies, apresentem $\mathrm{pH}$ e capacidade tampão adequados, osmolaridade compatível com o sêmen e devem proteger os espermatozoides contra as lesões criogênicas.

Os agentes crioprotetores são essenciais para o congelamento de quase todos os sistemas biológicos ${ }^{(7)}$ e devem ser adicionados aos diluentes para promoverem melhor sobrevivência espermática durante o processo de congelamento e descongelamento ${ }^{(5)}$. Desde a demonstração da sua eficácia ${ }^{\left({ }^{8}\right)}$, o glicerol é o agente crioprotetor mais utilizado na confecção de meios diluidores para conservação de sêmen ${ }^{(9)}$ e exerce função intracelular e extracelular ${ }^{(6)}$. No entanto, Fahy ${ }^{(7)}$ afirma que a atividade crioprotetora atribuída ao glicerol é prejudicada pela redução da viabilidade da célula espermática e fertilidade pósdescongelação.

Devido aos efeitos negativos do glicerol sobre o espermatozoide, algumas substâncias crioprotetoras estão sendo utilizadas como alternativa para os diluidores de congelamento, dentre elas as amidas ${ }^{(10)}$. Elas apresentam menor viscosidade e peso molecular quando comparadas ao glicerol, características 
que podem resultar em uma maior permeabilidade desses compostos na membrana plasmática, ocasionando menor estresse osmótico ao espermatozoide ${ }^{(11)}$.

O uso da dimetilacetamida (DMA) para conservação de sêmen foi relatado em diversas espécies com resultados satisfatórios, dentre elas: equinos ${ }^{(9,12)}$ aves $^{(13,14)}$ peixes $^{(15,16)}$ e suínos $^{(17,18)}$. Entretanto, na literatura mundial consultada, apenas um estudo avaliou a utilização da DMA para a criopreservação de sêmen ovino( ${ }^{(19)}$ e nenhum foi encontrado testando a associação DMA/glicerol. Dessa forma, o objetivo deste trabalho foi avaliar a dimetilacetamida em diferentes concentrações, associada ou não ao glicerol, sobre a viabilidade espermática do sêmen ovino pós-descongelação.

\section{Material e Métodos}

O presente estudo foi aprovado pela CEUA da Universidade Federal da Bahia, recebendo o número de protocolo $41 / 2014$.

O experimento foi realizado no Laboratório de Reprodução Animal da Universidade Federal da Bahia. Foram utilizados dez ejaculados de dois ovinos adultos, geneticamente superiores da raça Santa Inês, clinicamente sadios e com avaliação andrológica satisfatória ${ }^{(20)}$. Os reprodutores foram alimentados com dietas à base de feno, ração comercial para ovinos, água e sal mineral ad libitum.

Previamente ao início do estudo, os machos foram submetidos a duas colheitas diárias a cada dois dias por 15 dias, com objetivo de esgotar as reservas extragonadais. As colheitas de sêmen foram realizadas com a utilização de vagina artificial com água aquecida a $42{ }^{\circ} \mathrm{C}$. O ejaculado foi colhido em tubos graduados e protegidos da luz e da temperatura ambiente por um protetor térmico. Após a colheita, a amostra de sêmen foi encaminhada imediatamente para o laboratório de processamento e avaliada quanto aos parâmetros espermáticos descritos a seguir:

$\mathrm{O}$ volume do ejaculado foi verificado por pipetagem e registrado em microlitros $(\mu \mathrm{L})$. Através de uma gota de sêmen sobre uma lâmina pré-aquecida em mesa aquecedora a $37{ }^{\circ} \mathrm{C}$, observou-se o turbilhonamento (escala de 0-5) em microscopia óptica com aumento de 100x. Para observação do vigor (escala de 0-5), foi procedida a diluição (1:50) do sêmen no meio diluidor Tris-gema de ovo ${ }^{(21)}$. Na sequência, para avaliação de motilidade total e progressiva $(0-100 \%), 10 \mu \mathrm{L}$ desta amostra foram colocados entre lâmina e lamínula e observados em microscopia óptica com aumento de $200 \mathrm{x}^{(20)}$.

Para o cálculo da concentração espermática, empregou-se a câmara de Neubauer, com sêmen diluído na proporção de $10 \mu \mathrm{L}$ para $4 \mathrm{~mL}(1: 400)$ de solução de formol-salina tamponada ${ }^{(22)}$. A contagem foi realizada em microscopia óptica com aumento de 200x. O estudo da morfologia espermática foi realizado posteriormente através de preparação úmida à temperatura de $37^{\circ} \mathrm{C}$, colocando $10 \mu \mathrm{L}$ da amostra diluída sob lâmina e lamínula e observando os espermatozoides em microscopia de contraste de fase (aumento de 1.000X), computando e classificando 100 células de acordo com as anormalidades encontradas. Elas foram classificadas conforme descritas por Blom ${ }^{(23)}$.

Foram coletadas alíquotas para realização do teste supravital com corante eosina (EOS) ${ }^{(24)}$ e observação da morfologia espermática, classificando separadamente o percentual de caudas dobradas (CD). 
O choque osmótico (CO) foi realizado para avaliação da viabilidade funcional da membrana plasmática dos espermatozoides, incubando-se $10 \mu \mathrm{L}$ de sêmen em $500 \mu \mathrm{L}$ de água destilada por cinco minutos, sendo posteriormente fixado em $200 \mu \mathrm{L}$ de formol-salina tamponada. Em seguida, procedeu-se a contagem de 100 células em microscopia de contraste de fase com aumento de 1000x. $\mathrm{O}$ percentual de espermatozoides reativos ao $\mathrm{CO}$ foi determinado pela subtração do percentual de espermatozoides com cauda dobrada, verificados após o choque osmótico, pelo obtido na morfologia espermática com a eosina.

O ejaculado foi diluído em meio contendo Tris-gema de ovo acrescido de dois crioprotetores: o glicerol e a dimetilacetamida, sozinhos ou em associações, respeitando o limite máximo de 5\% da dimetilacetamida. Para tanto, foram formados sete tratamentos experimentais:

- GL6\%: o diluidor Tris-gema de ovo acrescido de glicerol a 6\%.

- DMA3\%: o diluidor Tris-gema de ovo acrescido de dimetilacetamida a 3\%.

- GL5\%+DMA1\%: o diluidor Tris-gema de ovo acrescido de glicerol a 5\% e dimetilacetamida a 1\%.

- GL4\%+DMA2\%: o diluidor Tris-gema de ovo acrescido de glicerol a 4\% e dimetilacetamida a 2\%.

- GL3\%+DMA3\%: o diluidor Tris-gema de ovo acrescido de glicerol a 3\% e dimetilacetamida a 3\%.

- GL2\%+DMA4\%: o diluidor Tris-gema de ovo acrescido de glicerol a $2 \%$ e dimetilacetamida a 4\%.

- GL1\%+DMA5\%: o diluidor Tris-gema de ovo acrescido de glicerol a 1\% e dimetilacetamida a 5\%.

Após a diluição do sêmen, foi realizado o envase em palhetas de $0,25 \mathrm{~mL}$, com uma concentração de $100 \times 10^{6}$ espermatozoides.

As palhetas de sêmen foram submetidas ao resfriamento e tempo de equilíbrio por 120 minutos em refrigerador com capacidade de $240 \mathrm{~L}$, sob curva de resfriamento de $0,47{ }^{\circ} \mathrm{C} / \mathrm{min}$. Quando então, foram distribuídas horizontalmente em plataforma que foram colocadas a $5 \mathrm{~cm}$ de altura da lâmina líquida de nitrogênio por 20 minutos. Em seguida as amostras foram mergulhadas no nitrogênio líquido $\left(-196^{\circ} \mathrm{C}\right)$, acondicionadas em raques devidamente identificadas e armazenadas em botijão de sêmen até o momento do descongelamento, obedecendo a um período mínimo de 24 horas de armazenamento.

As amostras dos diferentes grupos experimentais foram descongeladas em banho-maria a $38{ }^{\circ} \mathrm{C}$ por 50 segundos $^{(24)}$. Após o descongelamento, o sêmen de cada grupo era depositado em microtubos de $1,5 \mathrm{~mL}$, previamente aquecidos e mantidos a $37^{\circ} \mathrm{C}$, dos quais retiravam-se amostras para as análises in vitro. Para a avaliação subjetiva da motilidade (MT e MP), vigor espermático e teste supravital, procedeu-se como descrito para o sêmen in natura.

O choque osmótico (CO) também foi realizado como descrito para o sêmen in natura, entretanto a proporção foi modificada, incubando-se $10 \mu \mathrm{L}$ de sêmen em $100 \mu \mathrm{L}$ de água destilada por 5 minutos e na sequência a amostra foi fixada com $100 \mu \mathrm{L}$ de formol-salina tamponado ${ }^{(25)}$.

O delineamento estatístico empregado foi inteiramente casualizado ${ }^{(26)}$, no qual os ejaculados foram considerados as repetições $(n=20)$ e os diluidores de sêmen foram os tratamentos $(n=7)$. Processouse, separadamente, dez ejaculados por animal.

Para a análise estatística das características avaliadas, foi empregado o programa estatístico Statistical Analysis System (SAS) - versão 5.0. Assim, realizou-se a seguinte sequência de análises:

a consistência dos dados e a análise descritiva (médias e desvio-padrão) das características de 
interesse (MT, MP, Vigor, EOS e CO) foram realizadas mediante o emprego do Procedimento MEANS (PROC MEANS);

os parâmetros foram analisados quanto à normalidade da distribuição através do teste Shapiro-Wilk; as variáveis que apresentaram distribuição não normal foram submetidas ao teste de Kruskal-Wallis, para observação de possíveis diferenças entres os grupos experimentais. Quando essa diferença foi verificada, procederam-se o ajuste e a comparação entre os diferentes grupos através do teste de Bonferroni;

para as variáveis com distribuição normal, executou-se a análise de variância (ANOVA) utilizandose para tanto o Procedimento "General Linear Model" (PROC GLM);

e, para a comparação das médias, empregou-se o teste de Student-Newman-Keuls (SNK). Para todas as análises, foi estabelecido o nível de probabilidade de cinco por cento.

\section{Resultados}

As características do sêmen in natura dos ovinos submetidos ao estudo apresentaram-se dentro dos padrões mínimos exigidos pelo Colégio Brasileiro de Reprodução Animal ${ }^{(20)}$, com valores médios para motilidade total de $88,3 \%$, vigor de 3,6 e $88,4 \%$ de espermatozoides morfologicamente normais (Tabela 1).

Os resultados obtidos com a avaliação do sêmen in natura e pós-descongelamento dos diferentes tratamentos experimentais encontram-se descritos nas Tabela 1.

Tabela 1. Parâmetros da cinética espermática subjetiva e da viabilidade espermática no sêmen in natura e pósdescongelamento nos diferentes diluidores estudados (GL $6 \%$, DMA3 $\%$, GL5\%+DMA1\%, GL4\%+DMA $2 \%$, GL3\%+DMA3 $\%$, GL2 $\%+D M A 1 \%$, GL1\%+DMA $\%$ ) (Médias \pm desvios-padrão)

\begin{tabular}{|c|c|c|c|c|c|c|c|c|}
\hline \multirow[b]{2}{*}{ Parimetro } & \multicolumn{8}{|c|}{ TRATAMENTOS } \\
\hline & In nanure & GL6\% & DMA3 $\%$ & $\begin{array}{l}\text { GL5\%+ } \\
\text { DMA1 } \%\end{array}$ & $\begin{array}{l}\text { GL4\%+ } \\
\text { DMA2\% }\end{array}$ & $\begin{array}{l}\text { CL3\%+ } \\
\text { DMA3\% }\end{array}$ & $\begin{array}{l}\text { GL2\%+ } \\
\text { DMA4\% }\end{array}$ & $\begin{array}{l}\text { GL1\%+ } \\
\text { DMA5\% }\end{array}$ \\
\hline $\mathrm{MT}(\%)$ & $88,3 \pm 04,0 \mathrm{a}$ & $68,6 \pm 14,0 \mathrm{~b}$ & $53,0 \pm 16,3 \mathrm{~cd}$ & $60,3 \pm 11,5$ bcd & $73,6 \pm 11,7 \mathrm{ab}$ & $61,6 \pm 12,3$ bc & $47,8 \pm 10,5 \mathrm{~cd}$ & $45,3 \pm 15,6 \mathrm{~d}$ \\
\hline $\operatorname{MP}(\%)$ & $83,0=04,12$ & $62,6 \pm 14,3 b$ & $46,3 \pm 17,9 \mathrm{~cd}$ & $54,0 \pm 11,0$ bcd & $67,6 \pm 13,0 \mathrm{ab}$ & $55,6 \pm 12,3 \mathrm{bc}$ & $41,7 \pm 11,5 \mathrm{~cd}$ & $38,9 \pm 17,1 \mathrm{~d}$ \\
\hline VIG (1-5) & $3,6 \pm 00,3 a$ & $3,0 \pm 00,5$ bc & $2,6 \pm 00,4$ cde & $2,9 \pm 00,5 \mathrm{bd}$ & $3,3 \pm 00,4 a b$ & $2,7 \pm 00,3$ cde & $2,5 \pm 00,3 \mathrm{de}$ & $2,3 \pm 00,4 \mathrm{e}$ \\
\hline $\operatorname{EOS}(\%)$ & $78,9 \pm 09,5 \mathrm{a}$ & $32,3 \pm 15,2 \mathrm{bcd}$ & $32,6 \pm 13,2 \mathrm{bod}$ & $32,6 \pm 16,8 \mathrm{bcd}$ & $41,2 \pm 13,1 b c$ & $34,2 \pm 13,3$ bod & $29,8 \pm 14,0 \mathrm{bcd}$ & $22,5 \pm 15,8 \mathrm{~cd}$ \\
\hline $\mathrm{CO}(\%)$ & $38,9 \pm 16,3$ & $55,2 \pm 12,3$ & $49,4 \pm 19,0$ & $54,7 \pm 15,8$ & $49,1 \pm 16,5$ & $40,9 \pm 15,7$ & $43,5 \pm 15,5$ & $44,1 \pm 13,6$ \\
\hline DT (\%) & $11,6 \pm 06,7$ & $17,0 \pm 06,5$ & $19,1=09,8$ & $15,4 \pm 08,6$ & $18,2 \pm 06,9$ & $14,6=07,9$ & $15,2 \pm 06,2$ & $12,8=06,7$ \\
\hline $\operatorname{DM}(\%)$ & $05,6 \pm 04,7$ & $10,0 \pm 06,2$ & $10,8 \pm 08,8$ & $09,0 \pm 07,8$ & $09,6 \pm 07,8$ & $08,5 \pm 06,6$ & $08,6 \pm 06,1$ & $08,9 \pm 06,0$ \\
\hline
\end{tabular}
Motilidade total (MT), motilidade progressiva (MP), vigor espermático (VG).

Integridade estrutural da membrana plasmática (EOS, eosina), integridade funcional da membrana plasmática ( $C O$, formol-salina tamponada), defeitos totais (DT), defeitos maiores (DM).

Valores seguidos de letras minisculas distintas, na mesma linha, diferem entre si pelo teste $S N K(P<0,05)$.

A motilidade total (MT) pós-descongelamento dos grupos GL5\%+DMA1\%; GL4\%+DMA2\% e GL3\%+DMA3\%, foram semelhantes $(\mathrm{P}>0,05)$ ao tratamento controle (GL6\%). O diluidor GL4\%+DMA2\% foi o único que promoveu a manutenção da MT pós-descongelação, quando comparado com o sêmen in natura $(\mathrm{P}>0,05)$.

Assim como verificado para a MT, os tratamentos GL5\%+DMA1\%, GL4\%+DMA2\% e GL3\%+DMA3\% foram semelhantes $(\mathrm{P}>0,05)$ ao GL6\%, quanto a MP. O tratamento GL4\%+DMA2\% manteve a eficiência para a manutenção da cinética espermática, cujo MP não diferiu do sêmen in natura. 
Para o vigor espermático, pode-se chamar a atenção que o grupo controle (GL6\%) e o tratamento com $4 \%$ de glicerol associado a 2\% de DMA, foram os únicos que proporcionaram vigor espermático acima de 3,0, valor mínimo recomendado para sêmen ovino pós-descongelamento ${ }^{(20)}$.

Não foi encontrado diferença $(\mathrm{P}>0,05)$ entre os tratamentos avaliados pós-descongelamento, quanto a avaliação da integridade estrutural da membrana plasmática realizada (EOS).

O percentual dos espermatozoides reativos ao choque osmótico $(\mathrm{CO})$ não variou $(\mathrm{P}>0,05)$ entre os diferentes tratamentos experimentais. Também não houve variação $(\mathrm{P}>0,05)$ para defeitos espermáticos totais (DT) e maiores (DM).

\section{Discussão}

Os estudos que utilizaram a dimetilacetamida como crioprotetores seminais são escassos na espécie ovina. Bittencourt ${ }^{(19)}$ avaliou a viabilidade dos espermatozoides criopreservados com esta amida, em concentrações de 3\% e 6\% e comparou com o glicerol a 6\%. O autor observou que a DMA na concentração mais elevada promoveu efeito deletério importante sobre os parâmetros de MT, MP e supravital (EOS). Neste estudo, o sêmen criopreservado com concentrações de amida acima de 4\%, apesar de apresentarem valores decrescentes de MT, MP e EOS, as diferenças não foram significativas quando comparadas aos grupos com menores percentuais de DMA (GL5\%+DMA1\%). Entretanto, verificou-se, em ambos trabalhos, a superioridade do grupo controle GL6\% $(\mathrm{P}<0,05)$ frente aos diluidores que empregaram amida sozinha ou em elevadas concentrações.

Em trabalho mais recente, Vasquez et al. ${ }^{(27)}$ testaram em ovinos a DMA nas concentrações de 2,5\%, $5,0 \%$ e $10 \%$ em espermatozoides colhidos do epidídimo e mantidos a $4{ }^{\circ} \mathrm{C}$ por uma e três horas. Esses autores observaram que os parâmetros espermáticos de MP, EOS e CO não apresentaram diferenças significativas $(\mathrm{P}>0,05)$ entre a DMA nas diferentes concentrações e o controle (Tris-gema de ovo, sem crioprotetor ou com glicerol), exceto na DMA a 10\% em 1h de incubação.

O efeito deletério da DMA em concentrações acima de 4\%, verificados no presente estudo, discorda dos resultados encontrados por Medeiros et al. ${ }^{(9)}$, que utilizaram a DMA na concentração de 5\% para criopreservação de sêmen da espécie equina. Os autores observaram que, embora não tenha havido diferença significativa para a MT entre os diluidores com DMA e glicerol, a amida proporcionou valores numéricos superiores $(46,6 \%$ versus 27,9$)$. Os autores concluíram que essa variação da MT poderia ser explicada pelo fato de terem utilizado no estudo reprodutores com baixa tolerância ao glicerol.

O benefício da DMA em concentrações superiores também foi observado na criopreservação de sêmen em outras espécies. Em trabalho realizado com suínos, Bianchi et al. ${ }^{(17)}$ encontraram médias superiores $(\mathrm{P}<0,05)$ para DMA $(5 \%)$ em comparação ao glicerol $(3 \%)$, nas características de motilidade total $(53,8$ versus $38,1 \%)$ e integridade da membrana plasmática (50,9 versus 34,5\%), avaliados após a descongelação. Neste mesmo trabalho, a DMA não apresentou efeito deletério na concentração de 3 e 7\% para os mesmos parâmetros supracitados. Em outro estudo, Bianchi et al.(18) avaliaram a DMA a $5 \%$ e o glicerol a $3 \%$ sobre os parâmetros de MP, integridade estrutural da 
membrana plasmática e $\mathrm{CO}$, além das taxas de concepção e fertilização in vivo em suínos. Não foi observada diferença $(\mathrm{P}>0,05)$ entre os tratamentos avaliados, entretanto o sêmen congelado com DMA a 5\% obteve média 10\% superior em relação ao glicerol sobre a taxa de fertilização. Em estudo com aves, os melhores valores para os parâmetros espermáticos avaliados pós-descongelamento foram observados com utilização de DMA em concentrações de $6 \%{ }^{(14,28)}$. Dessa forma pode-se observar que, diferente de ovinos, os equinos, suínos e aves apresentaram melhores resultados pósdescongelamento em meios diluidores que utilizam a DMA em concentrações superiores, demonstrando variável tolerância dos espermatozoides aos diferentes níveis desse crioprotetor e sua específica toxicidade nas diferentes espécies.

Outros crioprotetores da função química amida foram avaliados para conservação de espermatozoides ovinos, dentre eles a dimetilformamida (DMF), metilformamida (MF) e acetamida. A eficácia da DMF foi estudada por Moustacas et al. ${ }^{(29)}$ e foi observada grande variação $(\mathrm{P}<0,05)$ na motilidade progressiva entre os tratamentos com DMF, isolada ou em associação com glicerol e o grupo controle (GL5\%). Esses autores concluíram que a DMF não apresentou benefícios sobre os espermatozoides submetidos a congelamento, mesmo na presença de glicerol na composição dos diluidores. Apesar de amidas distintas (DMA versus DMF), os achados do presente trabalho discordam dos relatados por Moustacas et al. ${ }^{(29)}$, já que foram obtidos resultados satisfatórios para os grupos com amida associada ao glicerol, respeitando o limite máximo de 3\% de DMA.

Graças et al. ${ }^{(30)}$ testaram a metilformamida (MF) em diferentes porcentagens (3, 5, 7 e 9\%) e avaliaram sua capacidade de proteção sobre o espermatozoide ovino submetido à criopreservação. $\mathrm{Na}$ etapa de pós-resfriamento, as médias encontradas para os parâmetros de MP e VIG foram similares $(\mathrm{P}<0,05)$ entre os tratamentos que continham MF e o grupo controle (GL5,3\%), contudo, após o descongelamento, taxas significativamente menores foram observadas nos grupos com MF, exceto a $3 \%$ para MP e 3 e $5 \%$ para VIG.

Utilizando a acetamida nos meios experimentais de congelação, Silva et al. ${ }^{(31)}$ observaram o efeito desse crioprotetor em concentrações de 3 e 5\% e comparou ao glicerol a 5\%. Para esses autores, os tratamentos com a amida foram incapazes de preservar a motilidade progressiva e as estruturas dos espermatozoides congelados, tornando as amostras inviáveis para inseminação artificial. Em outro trabalho utilizando acetamida ( $2 \%$ e $7 \%)$, Silva et al. ${ }^{(32)}$ encontraram médias significativamente menores de MP, VIG e integridade estrutural da membrana plasmática em relação ao diluidor com glicerol (GL5\%). Segundo os autores, o dano osmótico causado pela acetamida possivelmente foi a principal causa dos baixos índices de congelabilidade dos espermatozoides ovinos.

Através da literatura científica, fica claro que o glicerol é o crioprotetor mais utilizado e aceito para criopreservação de espermatozoide ovino ${ }^{(6,33,34)}$. Isso ocorre em decorrência de diversos fatores, dentre eles a composição da membrana plasmática dessa espécie, que possui elevada permeabilidade ao glicerol ${ }^{(2)}$, possivelmente relacionada às suas características químicas e peso molecular. Apesar da DMA fazer parte de grupo bioquímico diferente do glicerol, observa-se que ambos possuem peso molecular semelhantes, com $87,11 \mathrm{~g} / \mathrm{mol}$ e $92,02 \mathrm{~g} / \mathrm{mol}$, respectivamente, o que poderia justificar os resultados satisfatórios com a sua utilização para o sêmen ovino.

Na avaliação da integridade funcional da membrana plasmática, através do CO, não foram observadas diferenças $(\mathrm{P}>0,05)$ entre os grupos experimentais, seja com glicerol e amida sozinhos, seja com as diferentes associações. Resultados semelhantes para sêmen ovino foram encontrados por Vasquez et 
al. ${ }^{(27)}$, que testaram a DMA, e Jerez et al. ${ }^{(35)}$ com a DMF em um meio base contendo leite de soja desnatado associado ou não ao glicerol. Esses resultados se repetiram quando o estudo foi feito para a espécie caprina, em que diluidores com DMF e glicerol não diferiram quanto ao $\mathrm{CO}^{(36,37)}$.

A variação do efeito benéfico das diferentes amidas para a manutenção dos parâmetros espermáticos em ovinos pós-descongelação também foi observada para o $\mathrm{CO}$, já que, quando a amida utilizada foi a metilformamida, percentuais inferiores $(\mathrm{P}<0,05)$ de espermatozoides reativos ao teste hiposmótico foram obtidos em relação ao grupo contendo glicerol $^{(30)}$.

Não foram observadas diferenças $(\mathrm{P}>0,05)$ sobre os percentuais de defeitos totais (DT) e defeitos maiores (DM) nos diferentes tratamentos estudados, após o descongelamento. De acordo com Henry e Neves ${ }^{(20)}$, os valores máximos de DT e DM recomendados para espécie ovina são de, respectivamente, $20 \%$ e $10 \%$. Portanto, apenas o grupo contendo $3 \%$ de DMA ultrapassou discretamente o percentual de defeitos maiores (10,8\%), o que significa que, de uma forma geral, os diluidores e associações empregadas foram eficientes para a prevenção de alterações morfológicas importantes durante a criopreservação. Já Graças et al. ${ }^{(30)}$, ao utilizarem a MF para a criopreservação do sêmen ovino, não obtiveram o mesmo sucesso e apenas o tratamento com glicerol apresentou índice de alterações morfológicas dentro do preconizado para a espécie.

\section{Conclusão}

A dimetilacetamida em percentuais até 3\%, associada ao glicerol, mostrou-se eficaz para a manutenção da viabilidade espermática pós-descongelamento na espécie ovina. Entretanto, foi observado efeito deletério da DMA nas concentrações acima de $4 \%$ ou quando ela não foi associada ao glicerol no diluidor.

\section{Referências}

1. Aisen EG, Quintana M, Medina V, Morello H, Venturino A. Ultramicroscopic and biochemical changes in ram spermatozoa cryopreserved with trehalose-based hypertonic extenders. Cryobiology. 2005;50(3):239-249.

2. Salamon S, Maxwell WMC. Storage of ram semen. Animal Reproduction Science. 2000;62(1):77-111.

3. Leboeuf B, Restall B, Salamon S. Production and storage of goat semen for artificial insemination. Animal Reproduction Science. 2000;62(1):113-141.

4. Lima LF, Moura P, Passos PIB, Leal DR, Rumpf R, Neves JP. Influência de sistemas de refrigeração sobre a qualidade do sêmen ovino criopreservado em palhetas. Ciência Animal Brasileira. 2010;11(4):835-844.

5. Amann RP, Pickett BW. Principles of cryopreservation and a review of cryopreservation of stallion spermatozoa. Journal of equine veterinary Science. 1987;7(3):145-173.

6. Bittencourt RF, Oba E, Ribeiro Filho, AL, Chalhoub M, Azevedo HC, Bicudo SD (2013). Avanços na criopreservação do sêmen ovino I: diluidores e crioprotetores. Ciência Animal Brasileira. 2013;14(4):522-536.

7. Fahy GM. The relevance of cryoprotectant “toxicity” to cryobiology. Cryobiology. 1986;23(1):1-13. 
8. Polge C, Smith AU, Parkes AS. Revival of spermatozoa after vitrification and dehydration at low temperatures. Nature. 1949;164:666.

9. Medeiros ASL, Gomes GM, Carmo MT. Papa FO, Alvarenga MA (2002). Cryopreservation of stallion sperm using different amides. Theriogenology. 2002;58:273-276.

10. Alvarenga MA, Papa FO, Neto CR. Técnicas para incremento da qualidade do sêmen de garanhões. Revista Brasileira de Reprodução Animal. 2017;41(1):81-85.

11. Alvarenga MA, Papa FO, Landim-Alvarenga FC, Medeiros ASL. Amides as cryoprotectants for freezing stallion semen: a review. Animal reproduction Science. 2005;89(1):105-113.

12. Melo CM, Zahn FS, Martin I, Orlandi C, Dell'Aqua JA, Alvarenga MA, Papa FO. Influence of semen storage and cryoprotectant on post-thaw viability and fertility of stallion spermatozoa. Journal of equine veterinary Science. 2007;27(4):171-175.

13. Sood S, Malecki IA, Tawang A, Martin GB. Response of spermatozoa from the emu (Dromaius novaehollandiae) to rapid cooling, hyperosmotic conditions and dimethylacetamide (DMA). Animal Reproduction Science. 2011;129(1):89-95.

14. Mosca F, Madeddu M, Sayed AA, Zaniboni L, Iaffaldano N, Cerolini S. Data on the positive synergic action of dimethylacetamide and trehalose on quality of cryopreserved chicken sperm. Data in brief. 2016;9:1118-1121.

15. Varela Junior AS, Corcini CD, Gheller SMM, Jardim RD, Lucia T, Streit DP, Figueiredo MRC. (2012). Use of amides as cryoprotectants in extenders for frozen sperm of tambaqui, Colossoma macropomum. Theriogenology. 2012;78(2):244-251.

16. Alves JP, Corcini CD, Silva EF, Caldas JS, Cardoso TF, Piedras SR, Jardim RD, Varela Junior AS. The role of amides in seminal cryopreservation of wild silverside, Odontesthes bonariensis. Cryobiology. 2016;73(3):383-387.

17. Bianchi I, Calderam K, Maschio EF, Madeira EM, Rosa Ulguim R, Corcini CD, Bongalhardo DC, Corrêa EK, Lucia jr. T, Deschamps JC, Corrêa MN. (2008). Evaluation of amides and centrifugation temperature in boar semen cryopreservation. Theriogenology. 2008a;69(5):632-638.

18. Bianchi I, Calderam K, Maschio EF, Madeira EM, Rosa Ulguim R, Rambo G, Corrêa EK, Lucia jr. T, Deschamps JC, Corrêa MN. Inseminação artificial intrauterina em leitoas com sêmen criopreservado com dimetilacetamida e glicerol. Ciência Rural. 2008b;38(7):1978-1983.

19. Bittencourt RF. Viabilidade e fertilidade do sêmen ovino criopreservado com glicerol ou dimetilacetamida em meio com trealose e EDTA. [tese]. [Botucatu]: Faculdade de Medicina Veterinária e Zootecnia, Universidade Estadual Paulista Júlio de Mesquita Filho; 2009, 200 p.

20. Roberts SJ. Veterinary obstetrics and genital diseases (Theriogenology). 3.ed. Michigan: Edwards Brothers; 1986. 981p.

21. Hancoch JL. The morphology of boar espermatozoa. Journal of the Royal Microscopical Society. 1957;76:84-97.

22. Blom, E. The ultrastructure of some characteristic sperm defects and a proposal for a new classification of the bull spermiogram. Nordic Veterinary Medicine. 1973;25:383 - 391.

23. Cedenho AP, Tokunaga IM. Coloraçao supravital para espermatozoides: comparação entre as tecnicas de eosina e eosina-nigrosina / Comparison of supravital staining techniques for spermatozoa: eosin and eosinnigrosin. Jornal Brasileiro de Urologia. 1995;16(4):234-236.

24. Bittencourt RF. Viabilidade e fertilidade do sêmen ovino criopreservado com glicerol ou dimetilacetamida

Cienc. anim. bras., Goiânia, v.19, 1-10, e-48026, 2018 
em meio com trealose e EDTA. [tese]. [Botucatu]: Faculdade de Medicina Veterinária e Zootecnia, Universidade Estadual Paulista Júlio de Mesquita Filho; 2009, 200 p.

25. Menezes GFO, Bittencourt RF, Ribeiro Filho AL, Chalhoub M; Bittencourt MF, Oba E, Bicudo SD. Utilização do choque osmótico na avaliação da viabilidade de sêmen criopreservado de ovinos. Brazilian Journal of Veterinary Research and Animal Science. 2013;50(5):396-405.

26. Reis JC. Delineamentos experimentais. In: Estatística aplicada à pesquisa em ciência veterinária. Pernambuco: Independente; 2003. p.397-411. Português

27. Vásquez JH, Núñez VH, Florentini EA, Gonzales JM, Camargo LA, Valdivia ME. Effects of five cryoprotective agents on quality of sheep epididymal spermatozoa during pre-freezing. Livestock Science. 2013;152(1):94-99.

28. Zaniboni L, Cassinelli C, Mangiagalli MG, Gliozzi TM, Cerolini S. Pellet cryopreservation for chicken semen: Effects of sperm working concentration, cryoprotectant concentration, and equilibration time during in vitro processing. Theriogenology. 2014;82(2):251-258.

29. Moustacas VS, Cruz BC, Varago FC, Miranda DA, Lage PG, Henry M. (2011). Extenders containing dimethylformamide associated or not with glycerol are ineffective for ovine sperm cryopreservation. Reproduction in domestic animals. 2011;46(5):924-925.

30. Graças CP, Lim AIPG, Fidelis AAG, Cardoso JR, Blume H, Mondadori RG. Metil-formamida na criopreservação de sêmen ovino. Ciência Animal Brasileira. 2013;14(4):481-487.

31. Silva ECB, Cajueiro JFP, Silva SV, Vidal AH, Soares PC, Guerra MMP. In vitro evaluation of ram sperm frozen with glycerol, ethylene glycol or acetamide. Animal Reproduction Science. 2012a;132(3-4):155-158.

32. Silva ECB, Cajueiro JFP, Silva SV, Guerra MMP. Crioprotetores etileno glicol ou acetamida na viabilidade in vitro de espermatozoides congelados de ovinos. Ciência Rural. 2012b;42(6):1083-1088.

33. Salamon S, Maxwell WMC. Frozen storage of ram semen I. Processing, freezing, thawing and fertility after cervical insemination. Animal Reproduction Science. 1995;37(3-4):185-249.

34. Santos IW, Nóbrega Junior JE, Cesaro MP, Ilha GF, Rovani MT, Gonçalves PBD. Crioprotetor para sêmen de carneiro a base de plasma de gema mantém membrana acrossomal intacta após a descongelação. Ciência Rural. 2015;45(6):1107-1112.

35. Jerez R, González N, Olaciregui M, Luño V, Blas I, Gil L. Use of soy milk combined with different cryoprotectants for the ram semen cryopreservation. Small Ruminant Research. 2016;134:34-38.

36. Silva AF, Costa EP, Oliveira FA, Torres CAA, Silva Hass GT, Nascimento VA. Uso de dimetil-formamida associada ou não ao glicerol na criopreservação de sêmen caprino. Revista Brasileira de Zootecnia. 2006;35(2):452-456.

37. Bezerra FSB, Castelo TS, Alves HM, Oliveira IRS, Lima GL, Peixoto GCX, Bezerra ACSD, Silva AR. Objective assessment of the cryoprotective effects of dimethylformamide for freezing goat semen. Cryobiology. 2011; 63(3):263-266. 